\title{
FARMERS' EXPOSURE TO NOISE AND VIBRATION IN SMALL AND MEDIUM-SIZED FARMS
}

\begin{abstract}
Ričardas BUTKUS, Associated professor in the Institute of Agricultural Engineering and Safety, Faculty of Agricultural Engineering, Lithuanian University of Agriculture. Address: Studentu str. 15b, LT - 53362 Akademija, Kaunas distr., ricardas.butkus@asu.lt Gediminas VASILIAUSKAS, Associated professor in the Institute of Agricultural Engineering and Safety, Faculty of Agricultural Engineering, Aleksandras Stulginskis University, Lithuania. Address: Studentu str. 15b, LT - 53362 Akademija, Kaunas distr., gediminas.vasiliauskas@asu.lt (corresponding author)
\end{abstract}

\begin{abstract}
Occupational noise, hand-arm and whole-body vibration are the main human health risk factors in various economic activity sectors including agriculture. Workers of agricultural sector are usually under increased risk as their exposure to these risk factors is usually longer than reference 8 hours. Moreover, most agricultural activities are related with the processes which include multiple equipment and machinery therefore noise and vibration exposure analysis is a complex issue which is usually undeservedly simplified. This problem can be emphasized by statistical data provided by State Labour Inspectorate of the Republic of Lithuania. Occupational diseases registered for farmers, agricultural and forestry workers consist $16 \%$ of all those registered in Lithuania. Four of five occupational diseases registered in Lithuania are related to vibration and noise (musculoskeletal (66\%) and hearing loss (13\%) and has the increasing tendency over the last years. These tendencies demand a deeper analysis of noise and vibration exposure of farmers and farm workers as obtained results could help to specify the strategy or procedure to reduce negative exposure effects. The results reveal that noise exposure level usually exceed exposure action value of $80 \mathrm{dBA}$ while hand-arm and whole-body vibration exposure limit value of 1.15 and $5 \mathrm{~m} / \mathrm{s}^{2}$ respectively.
\end{abstract}

Keywords: exposure, hand-arm vibration, occupational noise, whole body vibration.

\section{INTRODUCTION}

It has been recognized over time that occupational noise, whole-body (WBV) and hand-arm vibration (HAV) are the major causes of discomfort for the drivers of various self-propelled machinery, operators of various hand-held equipment and stationary machinery. Usually workers of various economic activity sectors are affected by the finite number of noise or vibration sources in comparison with agricultural workers. This sector is especially sensitive to risk factors as typical duration and exposure does not limit with the reference working shift duration which is equal to 8 working hours. These reasons are the crucial factors, influencing high level of occupational diseases for agricultural workers. Statistical data provided by various sources generally provide similar information which shows that noise induced hearing loss (NIHL) and musculoskeletal diseases (caused by hand-arm or whole-body vibration) are dominant and usually consist more than $50 \%$ of those registered for the workers of various activity sectors, including agriculture.

Various risks and their effects on human health are usually determined by the measurement data which is obtained during the risk assessment procedure. For the agricultural subjects who have limited financial resources (typically small and medium farms) usually lack the information on noise and vibration levels. Because of this consequence, long-term exposure to occupational noise, vibration, dust or ergonomic stresses remains of unknown (but easily predictable) level which is usually above the safe limits. Workers of a small farms are also under the increased risk as usually exploit older agricultural machinery or hand tools, do not have enough knowledge or capabilities to implement technical noise or vibration reduction solutions or to use any kind of personal protection.

The scale of the problem can be represented with the data provided by Lithuanian Business Information Centre (2017). According to monthly updated information, in September 2017, majority of farms in Lithuania were small or medium sized. Approximately $60 \%$ of all farms registered in Lithuania have managed the area of arable land up to 5 hectares, while only 3.5\% - more than 30 hectares. This clearly show that analysis of various risk factors is of common interest as huge number of farmers might not manage the information of various stressors on their health. One of the issues is also that small farm workers are usually the family members therefore risk assessment procedure does not legally apply for such kind of workers.

Copyright (C) 2017 The Authors. Published by Aleksandras Stulginskis University. This is an open-access article distributed under the terms of the Creative Commons Attribution License (CC-BY 4.0), which permits unrestricted use, distribution, and reproduction in any medium, provided the original author and source are credited. 
There are lots of results provided by various researchers (Marvel, 1991; McBride, 2003; Solecki, 2003 and 2005) that agricultural workers not surprisingly are under the influence of occupational noise levels which can cause increased risk on their health. These studies also provide recommendations for noise level reduction, such as hearing conservation programs, the use of ear plugs or muffs which can help to reduce NIHL risk. However, this is usually not a solution as workers avoid using any personal protection despite of high noise levels. In the study of Humann (2005), noise dosimetry results were collected in swine farms. It was found that noise exposure level was above the exposure level of $85 \mathrm{dBA}$ while for some operations even higher (>90 dBA). These results agree with the findings of Beckett (2000) where noise levels can be as high as for: tractors 90.7 (range 75-102), milking area 76.4 (range 54-93), milking house 82.2 (range 50-99), vacuum pump 91.9 (range 70-105), milk cooling compressor 83.8 (range 50-100). In the previous study of Butkus et al. (2015) it was also found that noise levels are usually above the exposure action values in cereal farms. It was found that tractor age acts crucial role on operators' noise exposure as noise level in cab might increase by $1 \mathrm{~dB}$ over 1 year of exploitation. However, these results do not provide the full portrait of the full farmers' exposure to noise as usually does not include activities that are not directly related with agricultural production. This might include various works such as grass cutting with trimmers, brush cutting, usage of chainsaws or other hand-held tools which are usually not included to noise exposure assessment while have high noise level. Any other activities which have relatively short duration over the day (week) but high level, can cause significant increase in total noise exposure or noise dose. As an example of such activities can be the operation of any hand-held tools, impact drills for maintenance works, garden equipment and others which should be included to the calculation of noise exposure.

Other effects such as musculoskeletal diseases, white finger syndrome are caused by hand arm and whole body vibration. Agricultural workers are affected to human vibration as most activities include the machinery operation on uneven surfaces (which causes increased vibration risk). Solutions for the vibration reduction are more sophisticated and include the implementation of various technical solutions such as vibration isolation, reduction of disbalance and others. These means are usually costly and cannot be effectively reduced by any personal protection. As soil preparation works are most common in cereal farms, it was found by Futasuka et al. (2006) that vibration values might vary from $1.0 \mathrm{~m} / \mathrm{s}^{2}$ to $1.2 \mathrm{~m} / \mathrm{s}^{2}$ during ploughing operations, $1.8 \mathrm{~m} / \mathrm{s}^{2}$ to $2.2 \mathrm{~m} / \mathrm{s}^{2}$ when cultivating and $1.6 \mathrm{~m} / \mathrm{s}^{2}$ to $1.7 \mathrm{~m} / \mathrm{s}^{2}$ during the transport operations. In general, it can be concluded, that daily exposure of WBV and HAV is exceeded at workplaces of tractor drivers during most of agricultural operations. However, most of the human vibration studies of other authors are based on vibration exposure calculation for agricultural machinery or hand-held tools separately without integrating these results to overall exposure which is the main parameter for individual assessment.

The research goal was to collect noise and vibration data in small and medium-sized farms and to determine the noise and vibration exposure to agricultural workers.

\section{METHODOLOGY}

Assessment procedure used in this study was based on the requirements provided in the EU directive for occupational noise (2003/10/EC) and vibration (2002/44/EC). Noise and vibration measurements were collected in 15 farms and included various daily agricultural activities (tractor driving, various cultivation activities, transportation, various animal feed preparation works, grain harvesting) as well as other activities indirectly related to agricultural production (grass cutting with brush cutters, lawnmowers as well as using chainsaws, circular saws, angle grinders, impact drills, air impact wrenches etc.). Research was carried out in the total number of 14 small and medium sized farms.

Evaluation of occupational noise and vibration were based on the requirements of the above given directives. According to the EU Directive 2003/10/EC assessment of the occupational noise should be made in respect of the daily noise exposure levels $\left(L_{E X, 8 h}\right)$ and peak sound pressures $\left(p_{p e a k}\right)$. In this study we used the parameter $L_{E X, 8 h}$ for the evaluation of noise exposure which gives noise level normalized to reference 8 hour working shift. It should be stated that for most calculations total duration of various activities was longer in duration than typical eight hours. Exposure limit values and exposure action values in respect of the daily noise exposure levels and peak sound pressure $\left(L_{C, p e a k}\right)$ are given in the EU Directive 2003/10/EC as follows:

- exposure limit values: $L_{E X, 8 h}=87 \mathrm{~dB}(\mathrm{~A}), p_{\text {peak }}=200 \mathrm{~Pa}$ or $L_{C, \text { peak }}=140 \mathrm{~dB}(\mathrm{C})$;

- upper exposure action values: $L_{E X, 8 h}=85 \mathrm{~dB}(\mathrm{~A}), p_{\text {peak }}=140 \mathrm{~Pa}$ or $L_{C \text {,peak }}=137 \mathrm{~dB}(\mathrm{C})$;

- lower exposure action values: $L_{E X, 8 h}=80 \mathrm{~dB}(\mathrm{~A}), p_{\text {peak }}=120 \mathrm{~Pa}$ or $L_{C \text {,peak }}=135 \mathrm{~dB}(\mathrm{C})$.

Calculation of noise exposure is based on the integration of the sound pressure level which can be calculated as product of work time duration and noise level over the time period:

$$
L_{A, e q, T e}=10 \lg \left[\frac{1}{T_{e}} \sum_{i=1}^{n} t_{i} \cdot 10^{0,1 L_{\text {Aeq }, i}}\right], \mathrm{dBA}
$$

where: $\quad L_{A e q, T e}$ - equivalent A-weighted sound pressure level per measurement period $t_{i}$

$i$ - number of measurements. Total duration $t_{i}$ of the intervals is $T_{e}$.

$L_{E X, 8 h}$ is then derived from $L_{A, e q, T e}$ as follows: 


$$
L_{E X, 8 h}=L_{\text {Aeq }, T e}+10 \lg \left(\frac{T_{e}}{T_{0}}\right), \mathrm{dBA},
$$

where: $\quad L_{A e q, T e}-\mathrm{A}$-weighted sound pressure level over time period $T_{e}$;

$T_{0}$ - reference duration of 8 working hours.

Vibration exposure was evaluated as provided in EU Directive 2002/44/EC. Exposure action value for hand arm vibration is fixed at $A(8) \leq 2,5 \mathrm{~m} / \mathrm{s}^{2}$ while exposure limit value $A(8) \leq 5 \mathrm{~m} / \mathrm{s}^{2}$. Exposure values for whole body vibration are fixed at $0,5 \mathrm{~m} / \mathrm{s}^{2}$ and $1 / 15 \mathrm{~m} / \mathrm{s}^{2}$ respectively.

Vibration exposure value $A(8)$ was calculated as follows:

$$
A(8)=\sqrt{\frac{1}{T_{0}} \sum_{i=1}^{n}} a_{w i}^{2} T_{i},
$$

where: $\quad a_{w i}-$ frequency weighted acceleration average during operation $i$;

$T_{i}$ - duration of operation $i$ in seconds;

$T_{0}-$ work-shift duration in seconds (28 800s).

Noise was measured by using the class 1 noise level meters DeltaOHM 2010, Brüel\&Kjær 2250 and 2270 while vibration data was collected using the hand arm and whole-body vibration analyser Bruel \& Kjaer (B\&K) type 4447. Weighted RMS values of $\mathrm{x}, \mathrm{y}$ and $\mathrm{z}$ axis and total vibration acceleration $a_{h}$ were measured. Noise data included Aweighted noise level $L_{A, e q, T e}$. Measurements and assessment were carried out according to the requirements of international standards ISO 9612, ISO 5349-1, ISO 5349-2, ISO 2631-1 and ISO 2632-2.

\section{RESULTS}

Noise levels were measured for various tasks and are provided in Table 1. As seen from the data, sound pressure level might be as high as $80 \mathrm{dBA}$ for most tasks. For example, if the measured mean value for hand held tools for maintenance is $94,3 \mathrm{dBA}$, farmer will get the exposure level $L_{E X, 8}$ of $80 \mathrm{dBA}$ over the working time which is less than 20 minutes. It should be kept in mind that multiple tasks are present over the working day, therefore exposure level will increase significantly. Noise level of typical tasks for agriculture such as tractor or harvester driving, dairy farming is not amongst the noisiest works which means that exposure to noise is mainly affected by additional activities, such as machinery repair or environmental works. Another issue for increased noise risk on workers is the noise level of tractors and combine harvesters. This machinery in small farms was manufactured three or more decades ago, therefore noise levels might reach the values near to $100 \mathrm{dBA}$ (for tractors without cab) and to 96,5 dBA for combine harvesters. At the harvesting time, when the work shift is even longer than 8 hours, exposure of the combine harvester operator might reach approx. $100 \mathrm{dBA}$.

Table 1. Measured A-weighted SPL of various equipment and their noise levels

\begin{tabular}{|l|c|c|c|c|}
\hline Equipment & No. of items & Mean (dBA) & $\begin{array}{c}\text { Min } \\
(\mathrm{dBA})\end{array}$ & $\begin{array}{c}\text { Max } \\
(\mathrm{dBA})\end{array}$ \\
\hline Combine harvesters & 9 & 85,8 & 82,1 & 96,5 \\
\hline Tractors with cab & 12 & 87,3 & 82,5 & 95,8 \\
\hline Tractors without cab & 9 & 92,0 & 80,1 & 101,2 \\
\hline Hand held tools for machine maintenance & 9 & 94,3 & 77,5 & 104,6 \\
\hline $\begin{array}{l}\text { Hand held and guided tools for environmental works (lawnmowers, } \\
\text { brush cutters, chainsaws etc.) }\end{array}$ & 16 & 89,9 & 75,1 & 94,8 \\
\hline Grain equipment (augers, dryers, bin fans) & 9 & 86,2 & 79,2 & 90,3 \\
\hline Dairy farm equipment & 8 & 82,4 & 77,2 & 87,1 \\
\hline
\end{tabular}

Comparison between the noise level in farms of various profile show that noise level in dairy farming is slightly less than in grain farms. The noisiest activities in dairy farming is the mechanized milking (87.1 dBA) and noise caused by animals during feeding (82.1 dBA). These results agree with those obtained by Solecki (2005) and McBride (2003).

The results provided in Table 1 were analyzed further to define the number of works which exceed particular noise level. These results are given in Figure 1. The distribution curve shows that $74 \%$ of the measured values exceeded the level $L_{A, e q}>80 \mathrm{dBA}$, and more than $30 \% L_{A, e q}>90 \mathrm{dBA}$. Such results indicate that noise exposure $L_{E X, 8 h}$ will exceed the exposure action value if any task with the level of $>90 \mathrm{dBA}$ is performed longer than one hour which is very typical for most farmers. However, these results do not give the full view of noise exposure which is the product of number of tasks performed, duration of these tasks and noise level. Above mentioned levels are more or less in agreement with the findings of Humann et al. (2005) who found that yearly exposure level might reach 78.6 to $99.9 \mathrm{dBA}$. Such values might cause the noise induced hearing loss or hearing impairment. 


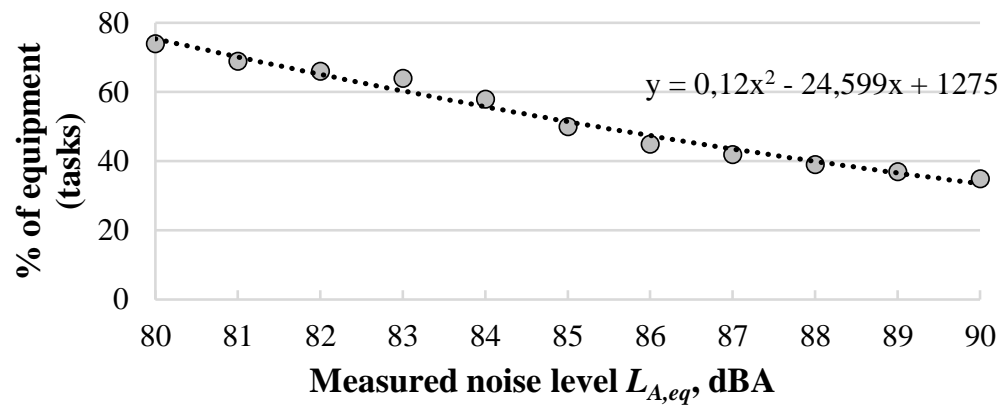

Figure 1. Percentage distribution of equipment (tasks) as a function of measured noise level

The above given results clearly show the necessity to use any technical, organizational or personal protective means for the noise reduction. As resources in small and medium farms are limited, one of the most effective and reasonable solutions is the use of personal hearing protection for the noisiest tasks at worst scenario. However, focusing interventions on just a few tasks or equipment with the highest noise level would diminish farmers' noise exposure, but unfortunately will not reduce the exposure to hazardous noise arising from any other activities of high level.

The second part of the research was related to the assessment of WBV and HAV operating various self-propelled, hand-guided and hand-held machinery. These results provided in the Figure 2 and include total number of 42 HAV measurements and $25 \mathrm{WBV}$ measurements. Acceleration values were grouped into frequency tables and histograms were constructed from the data. As seen in Figure 2a (HAV), majority (70 \%) of measured values lies within the interval of 2.5 to $5.0 \mathrm{~m} / \mathrm{s}^{2}$, while $>20 \%$ is even higher. This means that almost any combination of works for eight hour work shift will give the vibration exposure $A(8)$ value higher than exposure action value of $2.5 \mathrm{~m} / \mathrm{s}^{2}$ and credibly higher than $5 \mathrm{~m} / \mathrm{s}^{2}$ if the work duration is more than 8 hours. For the whole-body vibration case, the value of $0.5 \mathrm{~m} / \mathrm{s}^{2}$ is exceeded at more than $90 \%$ of the measured cases while $1.15 \mathrm{~m} / \mathrm{s}^{2}-$ at $35 \%$. Sources of hand arm vibration on farmers are generally related to operation of small hand-held tools, while whole body vibrations are mostly caused by agricultural machinery.

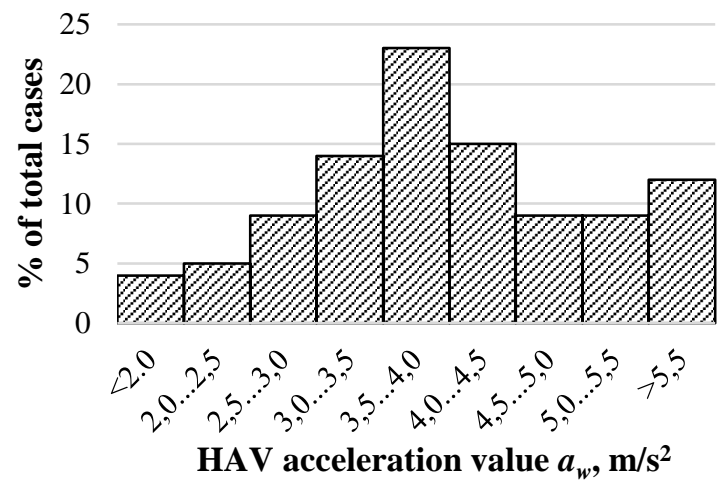

a)

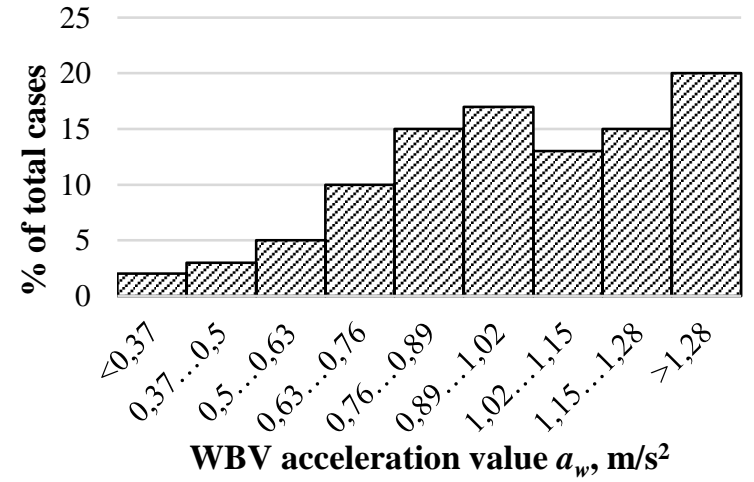

b)

Figure 2. Histograms of measured vibration acceleration values of various works in farms a) hand arm vibration $b$ ) whole body vibration

Values of hand-arm vibration were highest for air impact wrenches $\left(8.9 \mathrm{~m} / \mathrm{s}^{2}\right)$, chainsaws and angle grinders $\left(>5 \mathrm{~m} / \mathrm{s}^{2}\right)$, impact hammers $\left(>8 \mathrm{~m} / \mathrm{s}^{2}\right)$ while whole body vibration was highest for unsuspended tractors $\left(2.86 \mathrm{~m} / \mathrm{s}^{2}\right)$ during cultivation and combine harvesters $\left(2.19 \mathrm{~m} / \mathrm{s}^{2}\right)$.

The collected data could be used as a training material for the farmers in hearing or vibration conservation programs as reflects the situation in farms which is not usually estimated adequately by farmers. Educating farmers, their family members could significantly reduce negative noise and vibration effects as well as decrease occupational diseases.

\section{CONCLUSIONS}

1. Sound pressure level of $80 \mathrm{dBA}$ is exceeded in 3 out of 4 tasks in farms while the level of $90 \mathrm{dBA}$ in $>30 \%$ of tasks.

2. Most of the equipment operated in small farms might exceed the exposure action values as vibration acceleration for $90 \%$ of tasks is higher than $2.5 \mathrm{~m} / \mathrm{s}^{2}$ and $1.15 \mathrm{~m} / \mathrm{s}^{2}$ respectively.

3. Obtained results show the importance to constantly measure and evaluate occupational risk caused by noise and vibration. Depending on the risk, organizational and technical measures for noise and vibration reduction should be taken.

\section{REFERENCES}

1. Butkus, R., Liegus, M., Vasiliauskas, G. 2015. Tendencies of noise levels in cabs of agricultural tractors. Rural Development 2015: Towards the Transfer of Knowledge, Innovations and Social Progress: Proceedings of the 7th International Scientific Conference, 19-20th November 2015, pp. 1-5. 
2. EU Directive 2003/10/EC, On the minimum health and safety requirements regarding the exposure of workers to the risks arising from physical agents (noise), February 6, 2003.

3. EU Directive 2004/44/EC, On the minimum health and safety requirements regarding the exposure of workers to the risks arising from physical agents (vibration), June 25, 2002.

4. Futatsuka, M., Maeda, S., Inaoka, T., Nagano, M., Shono, M., Miyakita, T. 1998. Whole - body vibration and health effects in the agricultural machinery drivers. Industrial health, Vol. 36, pp. 127-132. https://doi.org/10.2486/indhealth.36.127

5. Humann, M.J, Donham, K.J., Jones, M.L., Achutan, C., Smith, B.J.2005. Occupational noise exposure assessment in intensive swine farrowing systems: dosimetry, octave band, and specific task analysis. Journal of Agromedicine, Vol. 10, No. 1, pp. 23-37. https://doi.org/10.1300/J096v10n01_04

6. Lithuanian Business Information Centre. Monthly report on farm size in Lithuania. Available at: http://www.vic.lt/uploads/file/1(51).pdf (Accessed at 25/10/2017)

7. Marvel, M. E., Pratt, D. S., Marvel, L. H. Regan, M., May, J.J. 1991. Occupational hearing loss in New York dairy farmers. American Journal of Industrial Medicine, Vol. 20, No. 4, pp. 517-531. https://doi.org/10.1002/ajim.4700200407

8. McBride, D. I., Firth, H. M., Herbison, G. P. 2003. Noise exposure and hearing loss in agriculture: a survey of farmers and farm workers in the Southland region of New Zealand. Journal of Occupational and Environmental Medicine, Vol. 45, No. 12, pp. 1281-1288. https://doi.org/10.1097/01.jom.0000100001.86223.20

9. Solecki L. 2003. Preliminary evaluation of occupational hearing loss risk among private farmers. The Annals of Agricultural and Environmental Medicine, Vol. 10, pp. 211-215.

10. Solecki L. 2005. Evaluation of annual exposure to noise among private farmers on selected family farms of animal production profile. The Annals of Agricultural and Environmental Medicine, Vol. 12, pp. 67-73. 